\section{Going live with aptamers}

\section{By Kai-Jye Lou, Staff Writer}

Researchers at the Duke University School of Medicine think they have improved upon a 20-year-old technology for selecting target-specific aptamers in vitro by making it work in vivo. ${ }^{1}$ Using the approach, the group identified an RNA aptamer that binds to a tumor-associated nuclear protein. The technique could simplify the generation of molecular probes and aid in target discovery and therapeutic development.

Aptamers are single strands of DNA or RNA that can bind to proteins and other molecular targets. Generation of tumor-targeting aptamers currently relies on in vitro approaches like SELEX (systematic evolution of ligands by exponential enrichment), in which aptamers from a library undergo iterative rounds of in vitro selection based on their ability to bind defined tumor proteins or whole cells. Researchers at the University of Colorado at Boulder developed SELEX about 20 years ago. ${ }^{2}$

"The fact that the identified
aptamer can localize to a
subcellular compartment
like the nucleus opens
the door to many future
developments."
-Craig Tuerk,
Morehead State University

"The in vivo SELEX-based strategy has been developed to generate a panel of aptamers that could be useful as molecular probes for revealing molecular-level differences between normal and tumor tissues and cells," said lead author Jing Mi, an assistant professor in the Department of Surgery at Duke. She said the approach also could identify aptamers that aid in the discovery of targets in other disease areas.

"The in vivo selection process is using the body as a filter to generate relevant RNA-based targeting molecules," said Bryan Clary, a corresponding author and chief of hepatopancreatobiliary and oncologic surgery at Duke.

Clary did have a caveat: because the selection process uses mice, the isolated aptamers may not bind to the human version of a target protein.

"We need to know if aptamers generated from the selection process in mice can also be used in humans," he told SciBX. "In this particular study, the aptamer we isolated does in fact bind to both human and murine versions of the protein, but this won't always be the case. It will be important to validate that targets identified in the mouse are also relevant in humans."

"In vivo imaging and targeting with aptamers is likely to be an area of great interest in the next decade because the reagents being developed keep getting better," said Larry Gold, who co-invented SELEX. Gold is chairman and CEO of SomaLogic Inc., which develops aptamer arrays to measure disease biomarkers in blood.

Gold founded NeXagen Inc. in 1991 to com-

The problem is that a target's conformation can vary between in vitro and in vivo settings. As a result, candidates isolated via in vitro selection may be unable to bind the same target in vivo. Also, in vitro nucleic acid selection approaches have a limited ability to account for off-target binding, and thus the selected aptamers may produce side effects.

To address both issues, a Duke group developed an in vivo analog of SELEX and used it to isolate tumor-specific RNA aptamers. The researchers took mice with metastatic colorectal cancer and injected them with a library of nuclease-resistant RNA molecules. Researchers then removed the tumors from the mice, extracted the RNAs that had homed to the tumors, amplified them and injected them into the next group of diseased mice.

Their rationale was that aptamers consistently found in the tumors following each round of selection should contain sequences that bind to tumor-specific targets.

After 14 successive rounds of in vivo selection, the lead RNA aptamer showed over a 70 -fold higher affinity for colorectal cancer protein extracts than for healthy colon proteins. The group showed that the aptamer bound to DEAD box polypeptide 5 (DDX5; p68), an RNA helicase that is predominantly found in the nucleus and is known to be upregulated in colorectal cancer. ${ }^{3}$

Results were published in Nature Chemical Biology. mercialize SELEX. In 1995 NeXagen and Vestar Inc. merged to become NeXstar Inc., which Gilead Sciences Inc. acquired in 1999. Gilead exclusively licensed SELEX to SomaLogic for diagnostic use in 1999 and to Archemix Corp. for therapeutic use in 2001.

\section{Unexpected benefits}

The ability of the Duke approach to find an aptamer that a priori made its way into a subcellular compartment could reduce the complexity of post-identification modifications that in vitro aptamers typically require.

"The observation that they identified aptamers that bind, with modest affinity, to a specific protein normally found in the cell nucleus was perhaps the most surprising and interesting finding of this study," Robert Schaub, SVP of discovery and development at Archemix, told SciBX.

"The fact that the identified aptamer can localize to a subcellular compartment like the nucleus opens the door to many future developments," said Craig Tuerk, the other co-inventor of the SELEX process and a professor in the Department of Biology and Chemistry at Morehead State University. "One could imagine using aptamers identified by similar in vivo screens on pathologic cells to piggyback therapeutic compounds or other aptamer sequences into the cell or to specific subcellular compartments. However, whether the observed results in 
the study are typical for other cancers and in other diseases remains to be seen."

One of the biggest differences between the in vitro and in vivo aptamer selection approaches is that the latter requires no knowledge of the aptamer's actual target. Indeed, Schaub noted that because in vivo selection relies on specific enrichment against unknown molecules on the cell surface, researchers are not selecting for aptamers that bind to a molecular target of their choosing.

"Detailed knowledge of molecular changes in the disease is not needed before the selection process, which could greatly simplify molecular probe development," Mi told SciBX.

On the other hand, Gold noted, "even if researchers know the aptamer binds to a tumor, they will still want to identify the aptamer's target. With the in vitro approach, the researchers know the protein they are targeting beforehand" because they are screening against a preparation of a purified protein. "Both approaches have value," he said.

"The in vivo process could have more value if an approach to select for specific targets could be integrated into it," said Archemix's Schaub.

Archemix's lead compound is ARC1779, an antiplatelet aptamer that targets von Willebrand factor (vWF). The compound is in a Phase IIb trial to treat thrombotic microangiopathies and a Phase IIa trial for the prevention of blood clots in patients undergoing carotid endarterectomy (CEA).
Clary said the Duke group is exploring the possibility of using the lead aptamer to deliver therapeutics to cancer cells but declined to disclose the specific payloads that are being evaluated. He added that the group also is repeating the in vivo selection process on a larger scale to discover additional aptamers.

Duke has filed for a patent covering the in vivo selection process and the reagents that are generated. The work is licensed to an undisclosed biotech.

Lou, K.-J. SciBX 3(1); doi:10.1038/scibx.2010.4

Published online Jan. 7, 2010

\section{REFERENCES}

1. Mi, J. et al. Nat. Chem. Biol.; published online Nov. 29, 2009; doi:10.1038/nchembio.277

Contact: Bryan M. Clary, Duke University School of Medicine, Durham, N.C.

e-mail: bryan.clary@duke.edu

Contact: Bruce A. Sullenger, same affiliation as above e-mail: bruce.sullenger@duke.edu

2. Tuerk, C. \& Gold, L. Science $\mathbf{2 4 9}$, 505-510 (1990)

3. Shin, S. et al. Cancer Res. 67, 7572-7578 (2007)

\section{COMPANIES AND INSTITUTIONS MENTIONED}

Archemix Corp., Cambridge, Mass.

Duke University School of Medicine, Durham, N.C.

Gilead Sciences Inc. (NASDAQ:GILD), Foster City, Calif.

Morehead State University, Morehead, Ky.

SomaLogic Inc., Boulder, Colo.

University of Colorado at Boulder, Boulder, Colo. 